\title{
Transcranial Direct Current Stimulation above the Medial Prefrontal Cortex Facilitates Decision- Making following Periods of Low Outcome Controllability
}

๑Dábor Csifcsák, Jorunn Bjørkøy, Sarjo Kuyateh, Haakon Reithe, and @Matthias Mittner https://doi.org/10.1523/ENEURO.0041-21.2021

Department of Psychology, UiT The Arctic University of Norway, Tromsø 9037, Norway

\section{Visual Abstract}

A Reinforcement learning task

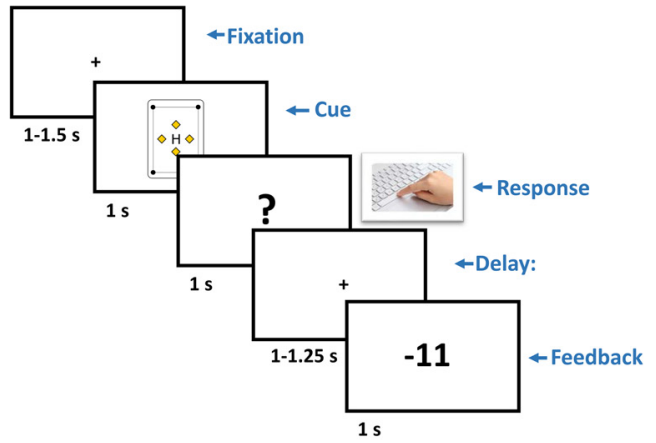

B HD-tDCS protocol

C Behavioral results

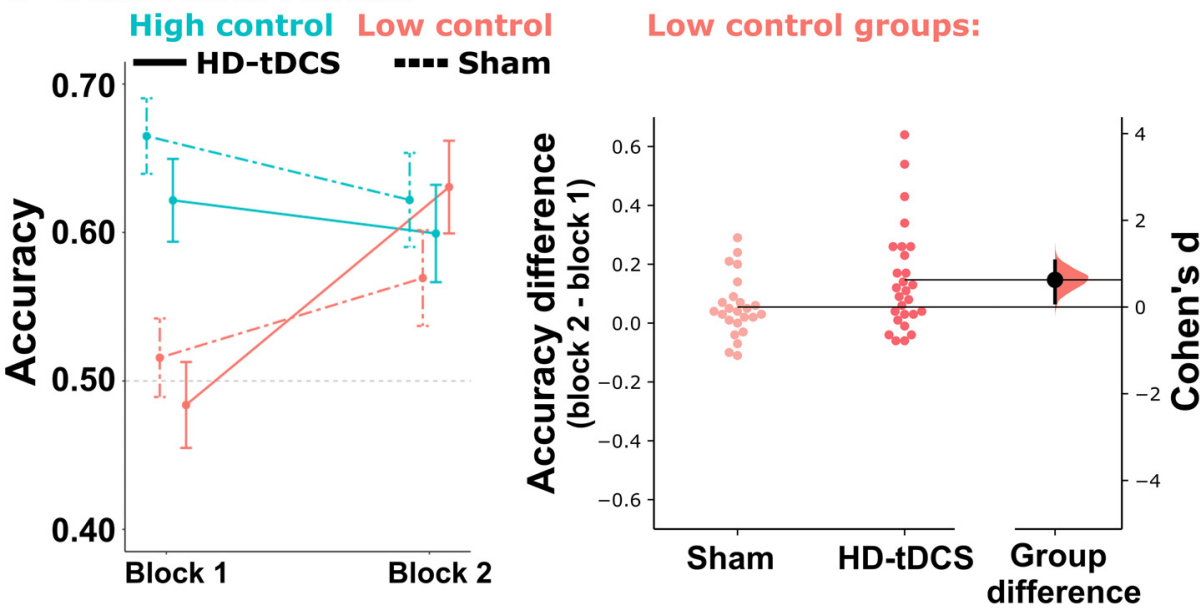

Recent studies suggest that choice behavior in reinforcement learning tasks is shaped by the level of outcome controllability. In particular, Pavlovian bias (PB) seems to be enhanced under low levels of control, manifesting in approach tendencies toward rewards and response inhibition when facing potential losses. The medial prefrontal cortex (mPFC) has been implicated both in evaluating outcome controllability and in the recruitment of cognitive control (CC) to suppress maladaptive PB during reinforcement learning. The current study tested 


\section{Significance Statement}

Our decisions are shaped by how much control we have over the situation. Under extreme circumstances, low controllability of choice outcomes can lead to learned helplessness (LH) and impaired coping. Since the medial prefrontal cortex (mPFC) was implicated in LH, we tested whether high-definition transcranial direct current stimulation (HD-tDCS) of this region counteracts the deleterious effects of low controllability of rewards and losses in healthy humans. We found stronger improvement in response accuracy when low controllability was combined with HD-tDCS. Moreover, several latent parameters of choice behavior were influenced by HD-tDCS and/or controllability. These results highlight the potential of our HD-tDCS protocol for interfering with choice arbitration in environments with reduced controllability, resulting in more adaptive behavior.

whether high-definition transcranial direct current stimulation (HD-tDCS) above the mPFC of healthy humans can influence PB, and counteract the previously documented, deleterious behavioral effects of low outcome controllability on decision-making. In a preregistered, between-group, double-blind study $(N=103$ adults, both sexes), we tested the interaction between controllability and HD-tDCS on parameters of choice behavior in a Go/NoGo task. Relative to sham stimulation, HD-tDCS resulted in more robust performance improvement following reduced control, an effect that was more pronounced in appetitive trials. In addition, we found evidence for weaker PB when HD-tDCS was administered during low controllability over outcomes. Computational modeling revealed that parameter estimates of learning rate and choice randomness were modulated by controllability, HD-tDCS and their interaction. Overall, these results highlight the potential of our HD-tDCS protocol for interfering with choice arbitration under low levels of control, resulting in more adaptive behavior.

Key words: decision-making; learned helplessness; medial prefrontal cortex; Pavlovian bias; reinforcement learning; tDCS

\section{Introduction}

Value-based decision-making is essential for guiding actions toward influencing external events in our favor. Recently, it has been suggested that deliberation strategies can be adjusted to the perceived level of controllability of the environment (Dorfman and Gershman, 2019; Ly et al., 2019). When uncertainty around action outcomes is increased, a commonly used heuristic is to rely more heavily on a Pavlovian bias (PB), manifesting in tendencies for approaching reward-predictive cues, and motor inhibition when facing potential punishment (Rangel et al., 2008; Dayan and Berridge, 2014). A possible explanation for this phenomenon is that the instrumental system relies on more effortful calculation of

Received January 29, 2021; accepted August 9, 2021; First published August $25,2021$.

The authors declare no competing financial interests.

Author contributions: G.C. and M.M. designed research; J.B., S.K., and H.R. performed research; G.C., J.B., S.K., H.R., and M.M. analyzed data; G.C. and M.M. wrote the paper.

This work was supported by the Northern Norway Regional Health Authority Grant PFP1237-15 (to G.C. and M.M.).

Acknowledgements: We thank Zsolt Turi for providing the card stimuli for the reinforcement learning task, Iselin Caroline Finn for her help in data collection, and Nya Mehnwolo Boayue for producing Figure $1 B$.

Correspondence should be addressed to Gábor Csifcsák at gabor. csifcsak@uit.no.

https://doi.org/10.1523/ENEURO.0041-21.2021

Copyright (C) 2021 Csifcsák et al.

This is an open-access article distributed under the terms of the Creative Commons Attribution 4.0 International license, which permits unrestricted use, distribution and reproduction in any medium provided that the original work is properly attributed. stimulus-action/action-outcome associations that does not pay off when outcomes are independent of actions. Conversely, Pavlovian stimulus-outcome learning may provide more precise predictions about upcoming events in the absence of response-feedback contingency, which in turn can optimize behavior more cost effectively (Dayan et al., 2006; Rangel et al., 2008; Dorfman and Gershman, 2019).

In extreme cases, the absence of control over aversive events can induce learned helplessness (LH), characterized by anxiety, motor passivity, and impaired decisionmaking (Pryce et al., 2011; Maier and Seligman, 2016). Once established, LH can also hinder problem solving in new situations with regained control, leading to persistent maladaptive coping. Intriguingly, inaction that is elicited by negative outcomes is a Pavlovian-type response (Rangel et al., 2008; Dayan and Berridge, 2014), raising the possibility that $\mathrm{LH}$ is an excessive manifestation of PB in decision-making (Maier and Seligman, 2016). However, the behavioral effects of $\mathrm{LH}$-induction might as well be because of passivity elicited by motor inhibition. To our knowledge, whether low outcome controllability influences Pavlovian response tendencies or whether it facilitates inaction in general, has not been investigated directly in animals.

On the neural level, perceived controllability has been associated with the medial prefrontal cortex (mPFC; Diener et al., 2010; Kerr et al., 2012; Maier and Seligman, 2016; Ly et al., 2019), which regulates activity in subcortical structures as a function of outcome controllability (Amat et al., 2005; Kerr et al., 2012; Maier and Seligman, 2016). In the case of LH, low perceived controllability of 
A

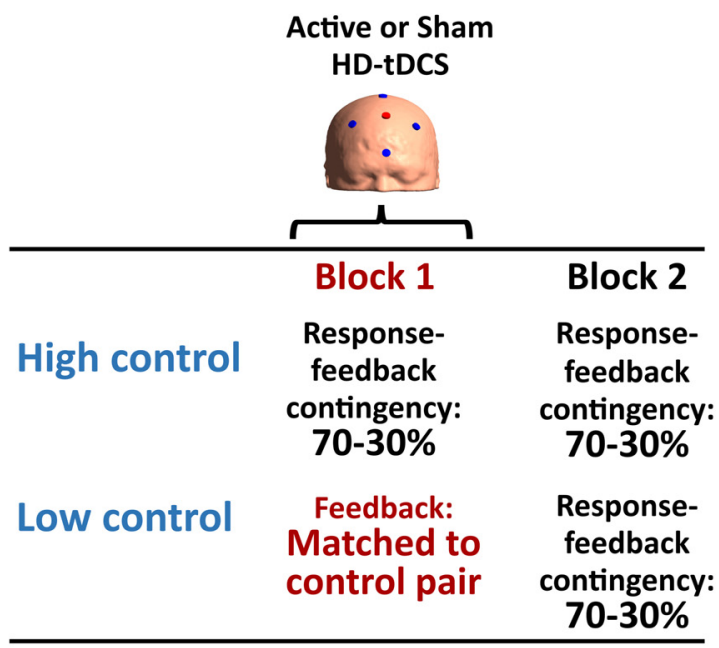

C

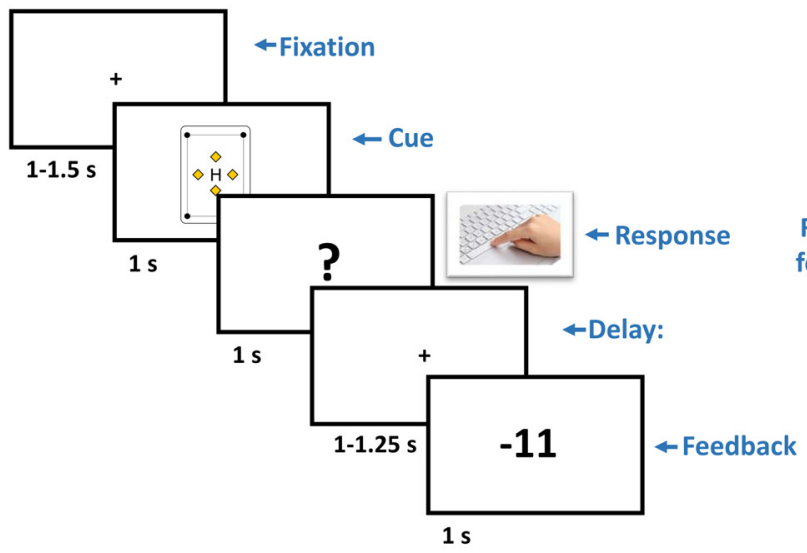

B

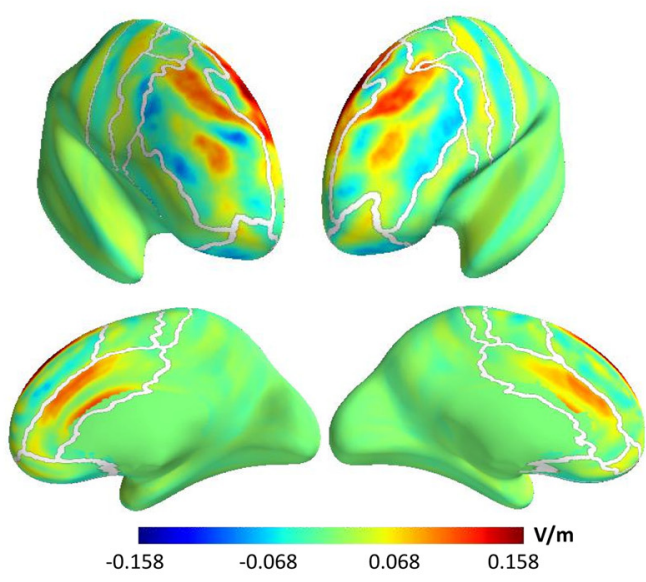

D

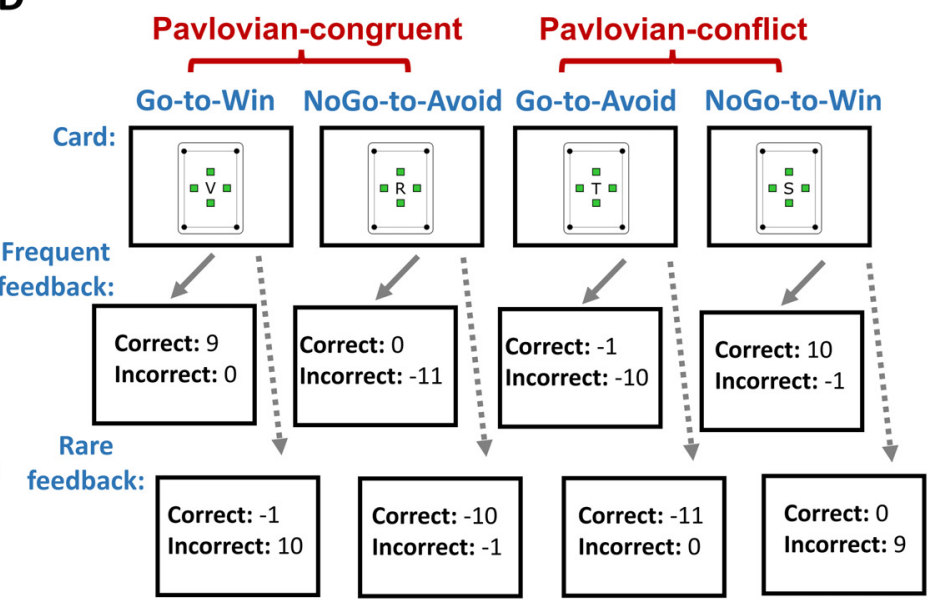

Figure 1. Overview of our study design $(\boldsymbol{A})$, the spatial distribution and magnitude of the normal components of HD-tDCS-induced electric fields, representing currents either entering or leaving the cerebral cortex (depicted with positive and negative values, respectively), averaged across 18 head models of healthy adults $(\boldsymbol{B})$, trial structure $(\boldsymbol{C})$, and card types with feedback values $(\boldsymbol{D})$.

negative events can lead to weaker top-down suppression from mPFC toward the dorsal raphe nucleus and the amygdala, both of which have been implicated in defensive behavior and pathologic responses to threat (Amat et al., 2005; Maier and Watkins, 2005; Kerr et al., 2012; Maier and Seligman, 2016; LeDoux and Daw, 2018).

The mPFC also seems to be crucial for mediating the balance between Pavlovian and instrumental responses. This is apparent under Pavlovian conflict, when Pavlovian and instrumental systems promote opposing action policies. For instance, avoiding an appetitive stimulus or approaching large losses can be difficult, since goal-directed aims are in conflict with Pavlovian response tendencies (Guitart-Masip et al., 2012; Hershberger, 1986; Huys et al., 2012). In these situations, cognitive control (CC) linked to the dorsal anterior cingulate cortex (dACC) was proposed as a mechanism for suppressing maladaptive PB, and consequently, to optimize behavior (Cavanagh et al., 2013; Cavanagh and Frank, 2014; Swart et al., 2018). A recent study provided a more direct link between PB, controllability and dACC activity, by showing that intermittent absence of control over rewards and losses during reinforcement learning enhanced $\mathrm{PB}$, and interfered with the neurophysiological correlate of CC, arising from dACC (Csifcsák et al., 2020). The authors concluded that manipulation of controllability levels can influence the magnitude of CC over PB in action selection.

The aims of the current study were twofold. First, using a new controllability manipulation, we wished to extend knowledge on the effect of low outcome controllability on PB and response accuracy during reinforcement learning (Dorfman and Gershman, 2019; Csifcsák et al., 2020). We hypothesized that, relative to a control condition, low controllability of outcomes would result in stronger PB and worse performance on Pavlovian-conflict trials. We anticipated that these effects, if sufficiently strong, would outlast the period of controllability manipulation, and manifest in a transfer to the subsequent block, where control over rewards and losses is restored.

Our second aim was to test whether high-definition transcranial direct current stimulation (HD-tDCS) above 
the MPFC reverses the behavioral consequences of low controllability (Fig. 1A). Whereas conventional tDCS montages use large electrodes that are placed further apart from each other, HD-tDCS consists of several, closely arranged small electrodes that provide more focal stimulation (Datta et al., 2009). We selected a " $4 \times 1$ " HD-tDCS protocol that provides, according to simulation studies (Csifcsák et al., 2018), relatively circumscribed and predominantly excitatory stimulation in the mPFC, potentially even reaching the dACC (Fig. 1B).

To investigate the interaction between controllability and HD-tDCS, we adopted a double-blind, between-group design following a preregistered protocol (https://osf.io/h45ju). Concerning our hypothesis about the effect of HD-tDCS, we predicted that, relative to the sham protocol, stimulation of the $\mathrm{mPFC} / \mathrm{dACC}$ would enhance CC, resulting in weaker PB and improved response accuracy both during and following HD-tDCS. In this respect, we postulated that HD-tDCS would counteract the deleterious effects of low outcome controllability on task performance.

\section{Materials and Methods}

\section{Participants}

Human subjects were recruited via public advertisements in Troms $\varnothing$, Norway. Based on our a priori power analysis [repeated-measures (rm)ANOVA, Cohen's $f=0.2$ for the within-between factor interaction for block $x$ group, $1-\beta=0.9, \alpha=0.05$, minimum required sample size $=96], 104$ healthy adults from both sexes signed the informed consent and were randomized to one of four groups differing in the level of outcome controllability (high vs low control) and HD-tDCS (stimulated vs sham). Data from one participant was discarded because of technical errors, yielding 103 participants (HighControl-Stimulated: $N=26$, HighControl-Sham: $N=26$, LowControl-Stimulated: $N=27$, LowControlSham: $N=24 ; 64$ females). We had to deviate from our prespecified exclusion criteria (i.e., excluding participants not producing at least one Go and one NoGo response to all four card types in both blocks) because an impractically high number of participants ( $N=50$, evenly distributed across groups) had to be excluded from further analysis using this criterion. This change was made before any formal data analysis took place and should therefore not compromise our preregistered analyses. A possible explanation for the high number of subjects not producing both response options to all cards in each block may be that 14 participants showed excessive PB (predominantly in block 1), whereas another 19 individuals could very successfully suppress their PB (mainly in block 2), leading to ceiling/floor effects in terms of response accuracy for some cards. However, we note that all participants in the final sample produced at least one Go and one NoGo response in both blocks. The groups did not differ in age $(\mathrm{M}=23.3$ years, $\left.\mathrm{SD}=2.7, F_{(3,99)}=0.61, p=0.606\right)$ or $\operatorname{sex}\left(H_{(3)}=2.08\right.$, $p=0.555)$. Participants received gift cards worth $\sim 22.5$ USD. The study protocol complied with the Declaration of Helsinki and was approved by the Institutional Ethics
Committee. All data and study materials are available at https://osf.io/d6eqk/.

\section{Study design}

First, a local anesthetic cream containing lidocaine/prilocaine ("EMLA") was distributed at electrode locations to ensure proper blinding. Next, we collected data on mood in the past month (PANAS-Past) and at the moment (PANAS-Present-Before; Watson et al., 1988), personality attributes of motivated behavior (BIS/BAS; Carver and White, 1994) and predisposition to develop hopelessness (BHS; Beck et al., 1974). Participants read task instructions (framed as a card game), performed a short practice session, and completed a quiz to ensure they understood all important aspects of the game. Quiz items with wrong answers were re-visited and discussed. Subsequently, we placed the electrode cap with electrodes and a small amount of conductive gel on the head of participants, and made sure that impedances were below $10 \mathrm{k} \Omega$.

The task consisted of two task blocks with a responsefeedback contingency of $70 / 30 \%$, except for block 1 in participants in the LowControl groups. Real or sham HDtDCS was also delivered during block 1 , using a prespecified double-blinded protocol. After each block, participants rated their perceived levels of success and control using two visual analog scales (data missing for one participant). At the end of the session, participants had to guess whether they received real or sham stimulation, which was followed by assessing momentary mood scores (PANAS-Present-After) and a working memory task (OSPAN; Turner and Engle, 1989).

\section{Task and controllability manipulation}

We used the modified version of the orthogonalized Go/ NoGo task that was designed to investigate the neural correlates of PB during instrumental learning (Cavanagh et al., 2013; Guitart-Masip et al., 2012). Participants had to collect points by learning whether to respond (Go: "pick up") or not (NoGo: "leave on the table") to each card. They were informed that there would be "winning" and "losing" cards, and that Win cards would either provide a reward (10 points) or zero outcome, whereas Avoid cards could result in a loss ( -10 points) or the absence thereof. Participants were also aware that favorable versus unfavorable outcomes were determined by correct versus incorrect responses, albeit in a probabilistic manner, with occasional "misleading outcomes." Outcomes were penalized by a "Go-cost" ( -1 point) if they were preceded by a Go response. Therefore, following an active response, win, no win/no loss and loss outcomes were modified to 9, -1 , and -11 points, respectively. The Go-cost was framed as the cost/effort of exploring by action, mimicking real-life situations (Teodorescu and Erev, 2014). The task consisted of two experimental blocks consisting of 160 trials each (four cards $\times 40$ repetitions).

For running the task, we used a desktop computer with Windows XP Professional operating system, Intel (R) Core (TM)2 Duo CPU, $2.33 \mathrm{GHz}, 1.96 \mathrm{~GB}$ RAM, and a 19-inch Sony Trinitron CRT monitor with $1024 \times 768$ resolution 
and $100 \mathrm{~Hz}$ refresh rate. Stimuli were presented and responses were collected using PsychoPy 1.83.04 (Peirce, 2007). Trials started with a central fixation sign, followed by a custom-made card, the response screen, a short delay and the outcome (Fig. 1C). Participants were asked to respond only when the question mark appeared on the screen, which always occurred $1 \mathrm{~s}$ after cue onset. Thus, the current task design did not enable assessing reaction times. In each block, four new cards were shown (Go-toWin, NoGo-to-Avoid, Go-to-Avoid, NoGo-to-Win), depending on their valence (reward vs loss) and action requirement (Go vs NoGo; Fig. 1D). Given that the Pavlovian system promotes approach toward rewards and inhibits response tendencies for losses, Go-to-Win and NoGo-to-Avoid cards were Pavlovian-congruent, whereas Go-to-Avoid and NoGo-to-Win cards were associated with Pavlovian conflict.

We aimed to induce helplessness by manipulating action-outcome contingency in the LowControl groups. Unbeknownst to the participants, each LowControl individual was paired with a HighControl participant. HighControl versus LowControl pairs were created by counterbalancing HD-tDCS conditions. For each HighControl participant, we recorded the outcomes from block 1 for the four card types separately, but removing the effect of the Go-cost when appropriate. These outcomes were shown in a random order, but in a card-specific manner to the corresponding paired subject in the LowControl group. That is, outcomes for a HighControl participant's Go-to-Win card were presented to the matched participant from the LowControl group for the card that was also labeled as Go-to-Win. Our manipulation ensured that controllability over rewards and losses was absent in this block (except for the Go-cost), while matching reward/loss frequency between groups. Importantly, manipulated outcomes were penalized by a Go-cost based on LowControl participants' own responses, leading to a possible discrepancy in reward/loss magnitude between HighControl and LowControl groups. For instance, a Go-associated reward (nine points) for a HighControl participant could be modified to 10 points if the same outcome was presented following a LowControl participant's NoGo response (or vice versa). By keeping the Go-cost during controllability manipulation we aimed for promoting behavioral passivity and limiting active exploration, which are key features of helplessness (Maier and Seligman, 1976; Teodorescu and Erev, 2014). However, it is important to note that the Go-cost provided some level of outcome controllability in block 1 to the LowControl groups, by reducing each outcome on active responding.

\section{HD-tDCS}

Brain stimulation was delivered with a Starstim device, using neoprene headcaps, conductive gel (SignaGel) and Ag/ $\mathrm{AgCl}$ electrodes with a diameter of $12 \mathrm{~mm}$ (Neuroelectrics). Electrodes were placed at scalp positions $\mathrm{Fpz}, \mathrm{Fz}, \mathrm{Cz}$, $F 3$, and F4, with Fz serving as anode (2 mA) and the surrounding four electrodes as returns ( $0.5 \mathrm{~mA}$ each). The choice of the electrode montage was based on our simulations of HD-tDCS-induced electric fields, using
18 realistic head models of healthy adults, downloaded from a freely available database (https://osf.io/ exbd5/; Boayue et al., 2018). Simulations were performed with the freely available SimNIBS software (Thielscher et al., 2015). We chose to evaluate the spatial distribution and magnitude of the normal component of electric fields, since it represents currents either entering or leaving the pial surface of the cortex, associated with predominantly excitatory or inhibitory effects (Rahman et al., 2013). Our montage yielded facilitatory currents in the superior-lateral and medial surfaces of the PFC in both hemispheres, possibly even reaching the dACC (Fig. 1B).

Real stimulation consisted of $30-s$ ramp-up, $15 \mathrm{~min}$ stimulation and 30-s ramp-down, whereas the sham session only contained two 30-s ramp-up/ramp-down periods at the beginning and end of a $16 \mathrm{~min}$ period, with no stimulation in-between. Neither the experimenters, nor the participants were aware of group assignment (double-blind protocols), and the percentage of participants guessing that they received real stimulation was comparable across groups, indicating proper blinding (HighControl-Stimulated: 34.6\%, HighControl-Sham: 50.0\%, LowControl-Stimulated: 55.5\%, LowControl-Sham: $60.8 \%$; $\left.H_{(3)}=3.84, p=0.279\right)$.

\section{Preregistered analysis}

Our primary focus was the change in PB across experimental blocks and groups. Therefore, we calculated the Pavlovian performance index (PPI) as the mean of two measures, reward-based invigoration (the number of Go responses on win trials/total number of Go) and punishment-based suppression (the number of NoGo responses on avoid trials/total number of NoGo). These indices represent the likelihood to initiate actions toward rewards and inhibit responses when facing potential loss, respectively (Cavanagh et al., 2013). We also calculated response accuracy as the ratio of correct responses for each block and card type.

PPI and accuracy were entered into rmANOVA with group as between-subject and block as within-subject factors, and additional within-subject factors of card congruency and valence for accuracy. Main effects and interactions were interpreted as significant at $p<0.05$. Estimates of effect size $\left(\eta_{\mathrm{p}}{ }^{2}\right)$ are also reported. Furthermore, Cumming estimation plots were used to illustrate effect sizes for pairwise comparison of conditions, whenever appropriate (Ho et al., 2019). We note, however, that Cumming estimation plots were not included in our preregistered analysis pipeline, and we used them to verify and/or extend results from rmANOVA. We chose estimation statistics because they provide robust estimates about the underlying effect sizes with resampling-based confidence intervals (Cls). Thus, this approach avoids pitfalls of dichotomous significance testing by focusing on the magnitude of effects, while also accounting for the precision of the estimation method using biascorrected and accelerated bootstrapping (Ho et al., 2019).

\section{Computational modeling}

To gain a more nuanced view on the effects of our interventions on latent processes of reinforcement learning 
and decision-making, we also implemented a computational model to our behavioral data. Previous studies on Pavlovian-instrumental interactions have successfully applied such models to extract various parameters of choice behavior, and showed that this approach unravels hidden associations that cannot be captured by more conventional data analysis (GuitartMasip et al., 2012; Cavanagh et al., 2013; Swart et al., 2018; Csifcsák et al., 2020). For computational modeling, we used a Precision 7920 Rack computer, Debian GNU/Linux 9.9 operating system, $2 \times$ Intel Gold 6152, $2.1 \mathrm{GHz}, 22$ cores, and $512 \mathrm{~GB}$ RAM. Our primary interest was to look for potential group differences in the temporal evolution of the PB parameter $\pi$, but we also extracted parameters representing randomness of choice (temperature; $\beta$ ), learning rate $(\alpha)$ and the general tendency to initiate actions (Go-bias; $b_{g o}$ ). This approach was very similar to those used in previous studies (Cavanagh et al., 2013; Swart et al., 2018; Csifcsák et al., 2020), with the exception that our model did not incorporate single-trial EEG data. All four parameters capture choice behavior from a different perspective. Changes in the Pavlovian parameter $(\pi)$ were expected to corroborate findings on PPI, with higher values in LowControl participants receiving sham stimulation, but a reduction during/following HD-tDCS. With respect to randomness of choice $(\beta)$, learning rate $(\alpha)$, and Go-bias $\left(b_{g o}\right)$, our analysis was more exploratory, aiming at providing supportive evidence to a study (Csifcsák et al., 2020) reporting increased values for all three parameters during manipulated outcome controllability.

Action choices (Go vs NoGo) for subject $i$ in trial $t$ of block $j$ for stimulus $s_{t}$ were modelled with the Bernoulli-experiment with probabilities $P(\mathrm{Go})$ and $P($ NoGo $)=1-p(\mathrm{Go})$ as

$$
\begin{gathered}
P\left(\mathrm{Go} \mid s_{t, j, i}\right)= \\
\frac{\exp \left[W_{t}\left(\mathrm{Goo} \mid s_{t, j, i}\right) / \beta_{j, i}\right]}{\exp \left[W_{t}\left(\mathrm{Go} \mid s_{t, j, i}\right) / \beta_{j, i}\right]+\exp \left[W_{t}\left(\text { NoGo } \mid s_{t, j, i}\right) / \beta_{j, i}\right]},
\end{gathered}
$$

where $W_{t}$ is response weight (Go vs NoGo) of the stimulus, and temperature parameter $\beta_{j, i}$ determines how biased the decisions are in favor of the higher-weighted option. For a given stimulus/action value $Q_{t}$,

$$
W_{t}\left(a \mid s_{t, j, i}\right)=\left\{\begin{array}{ll}
Q_{t}\left(\mathrm{Go} \mid s_{t, j i}\right)+b_{j, i}+\pi_{j, i} V\left(s_{t, j, i}\right) & \text { if } a=\mathrm{Go} \\
Q_{t}\left(\mathrm{NoGo} \mid s_{t, j, i}\right) & \text { if } a=\text { NoGo }
\end{array},\right.
$$

where parameter $b_{j, i}$ codes for a general Go-bias, and $\pi_{j, i}$ is our crucial PB parameter that scales learnt stimulus value $V\left(s_{t, j, i}\right)$ in a way that it favors action/inaction for win/avoid cards. The value of stimulus $s_{t, j, i}$ is cumulated as

$$
\left.V\left(s_{t, j, i}\right)=V_{t-1}\left(s_{t, j, i}\right)+\alpha_{j, i}\left[r_{t, i, j}-V_{t-1}\left(s_{t, j, i}\right)\right)\right],
$$

where $\alpha_{j, i}$ is the learning rate and $r_{t, j, i}$ is the reward (feedback). The final bit of the model is a standard Q-learning mechanism where stimulus/action pairs receive a value $Q_{t}\left(a \mid s_{t, j, i}\right)$ that are updated to the standard rule
Table 1: Statistical results for the comparison of questionnaire data and cognitive tests between the four experimental groups

\begin{tabular}{lllll}
\hline & \multicolumn{4}{c}{ Group effect } \\
Baseline measures & $F$ & $\mathrm{df}$ & $p$ & $\eta_{\mathrm{p}}{ }^{2}$ \\
\hline PANAS-Pa-Pos & 0.43 & 3,99 & 0.731 & 0.013 \\
PANAS-Pa-Neg & 0.17 & 3,99 & 0.916 & 0.005 \\
BIS/BAS & 0.49 & 3,99 & 0.691 & 0.015 \\
BHS & 0.46 & 3,99 & 0.710 & 0.014 \\
OSPAN & 0.14 & 3,96 & 0.933 & 0.004
\end{tabular}

BIS/BAS: behavioral inhibition/approach system; BHS: Beck hopelessness scale; OSPAN: operation span task; PANAS-Pa-Neg: negative past mood scores on the positive and negative affect schedule; PANAS-Pa-Pos: positive past mood scores on the positive and negative affect schedule.

$$
\left.Q_{t}\left(a \mid s_{t, j, i}\right)=Q_{t-1}\left(a \mid s_{t, j, i}\right)+\alpha_{j, i}\left[r_{t, j, i}-Q_{t-1}\left(a \mid s_{t, j, i}\right)\right)\right] .
$$

We model the data from all subject and sessions in the framework of hierarchical Bayesian modeling. We refer the reader to Gelman et al. (2003) for in-depth coverage of the advantages of this approach. All models where implemented using Hamiltonian Monte Carlo algorithms (Hoffman and Gelman, 2014) implemented in Stan (Carpenter et al., 2017). We used six parallel chains with warm-up period of 1000 samples each such that 6000 samples were drawn from the converged chains. Traceplots for all variables were manually screened for convergence. In addition, we calculated the GelmanRubin diagnostic (Gelman and Rubin, 1992) to ensure that all $R \leq 1.05$.

The dependency of each model parameter on block, controllability manipulation, HD-tDCS, and their interactions were included at the group-level in the hierarchical model directly. Posterior densities for the estimated coefficients were calculated and regarded as relevant if their 95\% highest density interval (HDI) excluded zero. When reporting regression coefficients, we report posterior mean $b, 95 \% \mathrm{HDI}$ and the evidence ratio (ER) in favor of a positive $\left(\mathrm{ER}_{+}\right)$or a negative effect (ER_). ER can be interpreted as an odds ratio, calculated as the ratio of two probabilities: the probability of the effect being positive, $P(b>0)$, divided by the inverse probability of the effect being zero or negative, $1-P(b>0)$, for $\mathrm{ER}_{+}$or its inverse for ER_. For example, the statement $b=0.09[0.01,0.18]$, $\mathrm{ER}_{+}=27.0$ indicates that it is 27 times as likely that the effect is positive than that it is zero or negative. In this analysis, $P$ values represent posterior probabilities that values are either below or above zero, and are not to be confused with frequentist $p$ values.

\section{Code accessibility}

The code/software described in the paper is freely available online at https://osf.io/d6eqk/.

\section{Results}

\section{Preregistered analysis}

Statistical analysis of questionnaire data and working memory performance collected at baseline are presented in Table 1. Experimental groups did not differ in their past mood (PANAS-Past), hopelessness (BHS), personality 
Table 2: Statistical results for the comparison of questionnaire data and subjective ratings between the four experimental groups and the repeated measurements

\begin{tabular}{|c|c|c|c|c|c|c|c|c|c|c|c|c|}
\hline & \multicolumn{4}{|c|}{ Group effect } & \multicolumn{4}{|c|}{ Block effect } & \multicolumn{4}{|c|}{ Block $\times$ group interaction } \\
\hline & $F$ & df & $p$ & $\eta_{\mathrm{p}}^{2}$ & $F$ & df & $p$ & $\eta_{\mathrm{p}}^{2}$ & $F$ & df & $p$ & $\eta_{\mathrm{p}}^{2}$ \\
\hline PANAS-Pr-Pos & 0.37 & 3,98 & 0.820 & 0.009 & 23.99 & 1,98 & $<0.001$ & 0.197 & 1.75 & 3,98 & 0.162 & 0.05 \\
\hline PANAS-Pr-Neg & 0.61 & 3,98 & 0.611 & 0.018 & 10.99 & 1,98 & 0.001 & 0.101 & 1.33 & 3,98 & 0.267 & 0.04 \\
\hline Success rating & 0.12 & 3,98 & 0.949 & 0.004 & 12.27 & 1,98 & $<0.001$ & 0.111 & 2.39 & 3,98 & 0.073 & 0.07 \\
\hline Control rating & 0.81 & 3,98 & 0.488 & 0.024 & 0.80 & 1,98 & 0.373 & 0.008 & 0.81 & 3,98 & 0.492 & 0.024 \\
\hline
\end{tabular}

PANAS-Pr-Neg: negative momentary mood scores on the positive and negative affect schedule; PANAS-Pr-Pos: positive momentary mood scores on the positive and negative affect schedule. Significant $(p<0.05)$ effects are highlighted with bold.

traits (BIS/BAS), or working memory capacity (OSPAN). Statistical results concerning repeated measurements of momentary mood ratings (PANAS-Present) revealed reduced scores by the end of the session, but no group differences were found (Table 2). Subjective ratings of perceived success increased by block 2 , but scores were statistically comparable across groups (Table 2; Fig. 2A). As for perceived outcome controllability, no main effects or interactions were found (Table 2; Fig. 2B).

With respect to our PB measure, PPI, a significant reduction in block 2 confirmed that participants gradually learned to suppress their PB (block: $F_{(1,99)}=3.99, p=$ $0.048, \eta_{p}{ }^{2}=0.039$ ). However, PPI was not influenced by group membership, despite showing lower values in the crucial LowControl-Stimulated group (group: $F_{(3,99)}=$ 2.11, $p=0.104, \eta_{\mathrm{p}}{ }^{2}=0.060$; block $\times$ group: $F_{(3,99)}=0.14$, $p=0.937, \eta_{\mathrm{p}}{ }^{2}=0.004$; Fig. $3 A$ ). Paired data from all individuals along with the effect size estimates (bootstrapped 95\% Cls) for changes in PPI from block 1 to block 2 are shown in Extended Data Figure 3-1, indicating comparable Block-effects across the four groups. Crucially, Cumming estimation plots for the effect size (Cohen's $d$ ) obtained for the comparison of PPI values from the three groups receiving experimental interventions (HighControl-Stimulated, LowControl-Sham, LowControl-Stimulated) against a shared control (HighControl-Sham) showed reduced PPI only in the LowControl-Stimulated group in block 1 , quantified by a medium mean effect size (mean = $-0.63,95 \% \mathrm{Cl}=[-1.13,-0.01]$; Fig. 3B). A similar trend was observed in block 2, although the $95 \% \mathrm{Cl}$ did not exclude zero $(-0.52$ [-1.09, 0.05]; Fig. 3C). Finally, we assessed whether the two sub-measures of PPI, reward-based invigoration and punishmentbased suppression were similarly influenced by our interventions. This analysis yielded largely similar results to PPI (estimates for Cohen's $d$ for the LowControlStimulated vs HighControl-Sham comparison: rewardbased invigoration in block 1: $-0.59[-1.1,-0.06]$, block 2: -0.48 [-1.02, 0.08], punishment-based suppression in block 1: $-0.62[-1.12,-0.09]$, block 2: $-0.55[-1.1,0.05])$. For all other comparisons, mean effect size estimates were substantially weaker (between -0.27 and -0.04 ), and $95 \%$ Cls always included zero. Overall, these findings provide some evidence for the efficacy of HD-tDCS in reducing PB when mPFC stimulation occurs simultaneously with the absence of control over rewards and losses.

Analysis of response accuracy revealed significant main effects for congruency $\left(F_{(1,99)}=16.60, p<0.001\right.$, $\left.\eta_{\mathrm{p}}{ }^{2}=0.144\right)$, block $\left(F_{(1,99)}=5.72, p=0.019, \eta_{\mathrm{p}}{ }^{2}=0.055\right)$, and group $\left(F_{(3,99)}=4.52, p=0.005, \eta_{\mathrm{p}}{ }^{2}=0.120\right)$. Importantly, the significant block $\times$ group interaction $\left(F_{(3,99)}=9.60\right.$, $p<0.001, \eta_{\mathrm{p}}^{2}=0.225$ ) was because of significantly improved responding from block 1 to block 2 in the LowControlStimulated group only $\left(p_{\text {Bonferroni }}<0.001 ; p_{\text {Bonferroni }}>0.068\right.$ for other groups; Fig. $4 A$ ), with only this group showing increased mean response accuracies by block 2 for all four card types (Extended Data Fig. 4-1). As a result of low
A

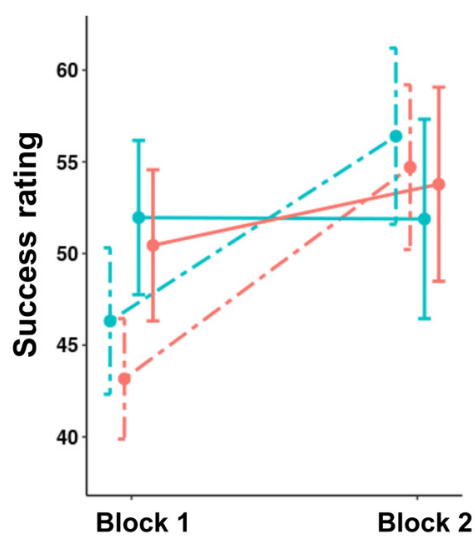

B

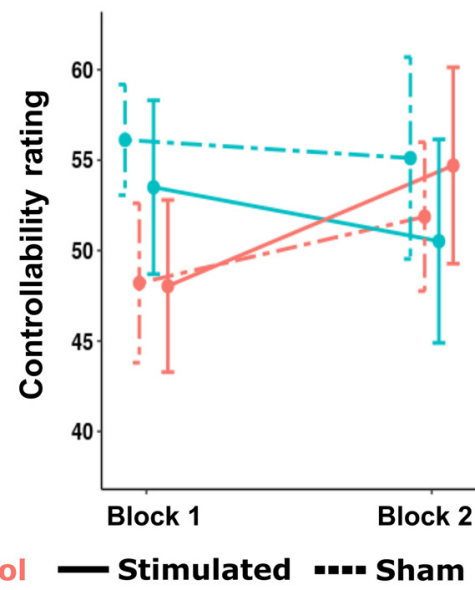

Figure 2. Ratings (means and SEs) of perceived success $(\boldsymbol{A})$ and outcome controllability $(\boldsymbol{B})$ following each block. 
A

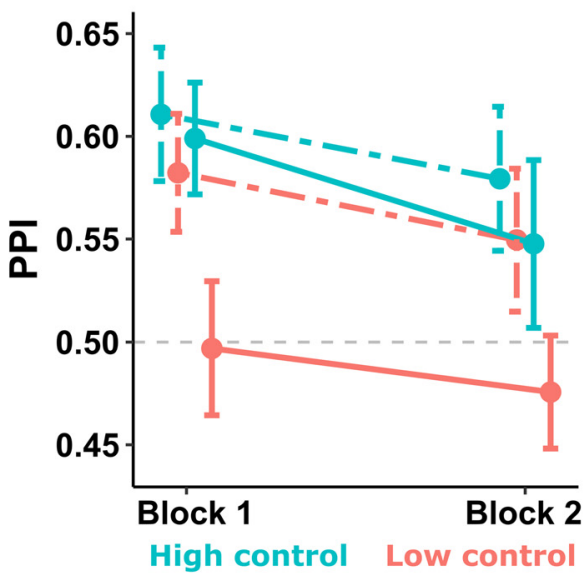

High control Low control
B
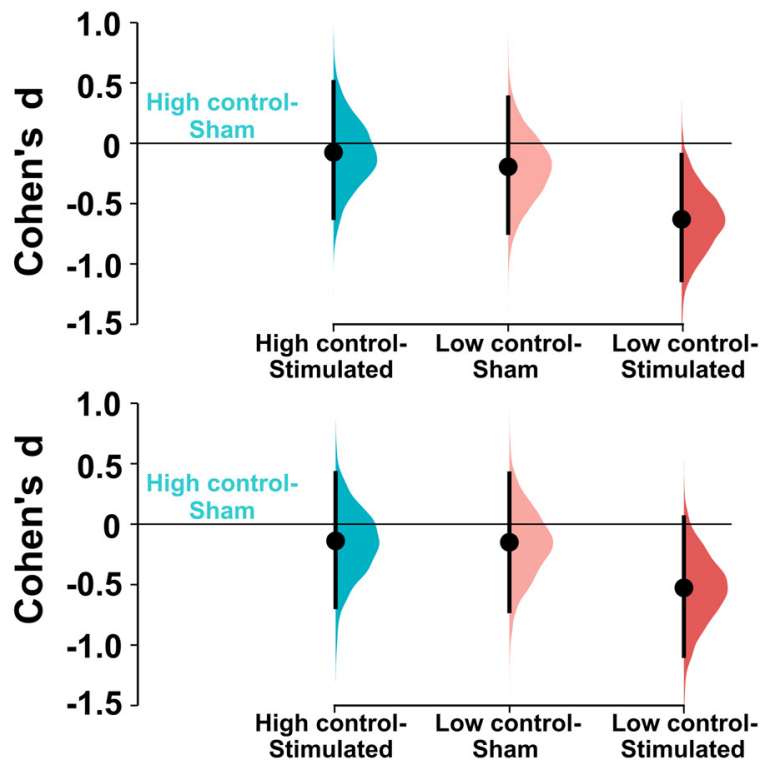

Figure 3. Changes in the magnitude of the PPI (means and SEs) across the two blocks and four experimental groups (A), and Cumming estimation plots showing effect size estimates (Cohen's $d$ ) for three comparisons against a shared control condition (HighControl-Sham group) for block $1(\boldsymbol{B})$ and block $2(\boldsymbol{C})$. Mean differences are presented as black dots, along with the corresponding bootstrap sampling distributions (5000 samples) and the bias-corrected and accelerated $95 \%$ Cls (black bars). Raw data and Cumming estimation plots related to changes from block 1 to block 2 for each group are presented in Extended Data Figure 3-1.

controllability, both LowControl groups produced significantly worse response accuracies in block 1 relative to HighControl groups ( $p_{\text {Bonferroni }}<0.023$ for all comparisons; Fig. $4 A$ ).

When comparing changes in response accuracy between blocks using Cumming estimation plots, we found effect size estimates with the $95 \% \mathrm{Cl}$ excluding zero for both LowControl groups (HighControl-Sham: -0.27 $[-0.75,0.09]$, HighControl-Stimulated: -0.16 [-0.42, 0.06], LowControl-Sham: 0.55 [0.14, 0.94], LowControlStimulated: 1.17 [0.73, 1.61]; Extended Data Fig. 4-2). To verify that this effect was larger in the LowControlStimulated than in the LowControl-Sham group, we calculated block 2 minus block 1 difference scores, and estimated effect sizes for the stimulated versus sham HD-tDCS comparison, separately in the HighControl and LowControl conditions (Fig. 4B). This analysis revealed negligible effect for HD-tDCS in the HighControl groups $(0.15[-0.39,0.65])$, but a medium mean effect size in the LowControl-Stimulated versus LowControl-Sham comparison $(0.63[0.10,1.06])$, confirming results from rmANOVA (Fig. 4A). This effect was robust against removing one participant from the LowControl-Stimulated group, who was the only one in the whole sample producing accuracy scores of both $<0.3$ and $>0.7$ in the two blocks, resulting in an extremely large difference score (Cohen's $d$ for the comparison of LowControl groups after exclusion: 0.56 [0.003, 1.06]).

The preregistered rmANOVA also indicated a significant congruency $\times$ valence $\times$ group interaction $\left(F_{(3,99)}=3.49\right.$, $p=0.019, \eta_{p}{ }^{2}=0.096$; Fig. $4 C$ ). Here, all groups responded more accurately to Pavlovian-congruent cards in the loss domain (NoGo-to-Avoid > Go-to-Avoid; $p_{\text {Bonferroni }}<0.003$ ), an effect that was consistent across both blocks (Extended Data Fig. 4-3). For rewarding cards, however, no clear effect of congruency was found $\left(p_{\text {Bonferroni }}>0.099\right)$, except for the LowControl-Stimulated group, where performance was surprisingly worse for Pavlovian-congruent Go-to-Win versus conflicting NoGo-toWin cards ( $p_{\text {Bonferroni }}=0.008$ ). Consequently, accuracy was comparable between groups for all Avoid cards as well as in conflicting NoGo-to-Win trials $\left(p_{\text {Bonferroni }}>0.074\right.$ ), but for Pavlovian-congruent Go-to-Win cards, the LowControlStimulated group's performance was worse than that of HighControl-Stimulated $\left(p_{\text {Bonferroni }}=0.009\right)$ and HighControlSham participants $\left(p_{\text {Bonferroni }}=0.002\right)$. The paradoxical effect of improved responding to Pavlovian-conflict Win cards in LowControl participants was most pronounced in LowControl-Stimulated participants in both blocks (Extended Data Fig. 4-3). While in block 2, this was because of improved responding to conflict NoGo-to-Win cards, in block 1 , the effect was driven by reduced accuracy for congruent Go-to-Win cards. Given that controllability manipulation in the first block invalidates the concept of Pavlovian congruency (out of the two Win and two Avoid cards, one is arbitrarily labeled as Go and one as NoGo, but these attributes cannot be learned in the absence of action-outcome contingency), the number of Go responses must necessarily be similar for congruent versus conflict cards in this block. To verify this, we also calculated the percentage of Go responses for each card type and block. Indeed, the main effect of Congruency was not significant for block 1 in LowControl groups either for Win or Avoid cards $(F<1.43, p>0.237)$, while it was significant for HighControl groups in block 1 and all four groups in block 2, in both the gain and loss domains (all $F>12.75, p<0.001$; Extended Data Fig. 4-4). 


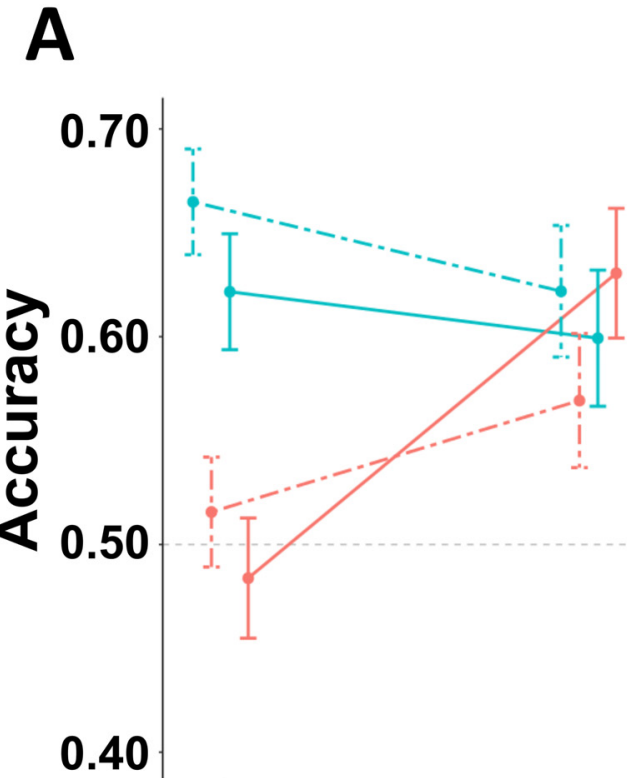

Block 1
C

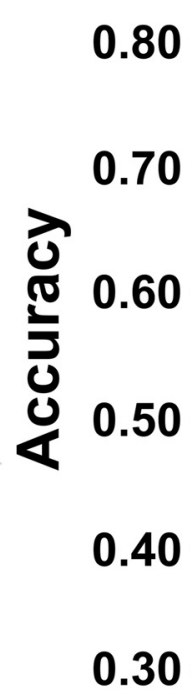

Avoid cards:

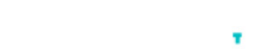

Win cards: -
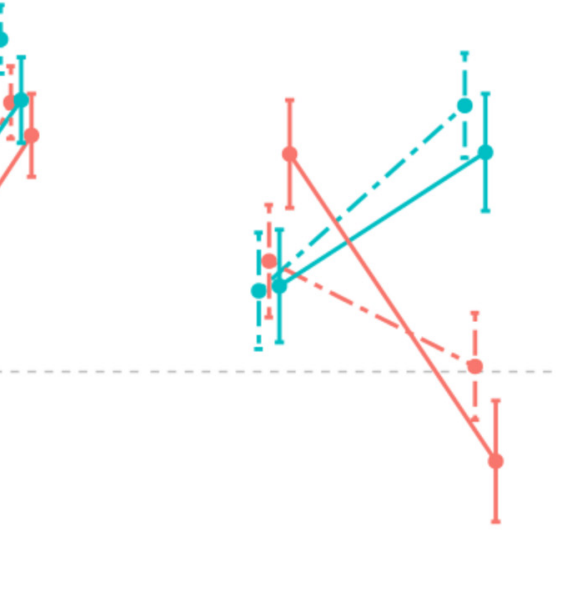

\section{High control Low control - Stimulated m... Sham}
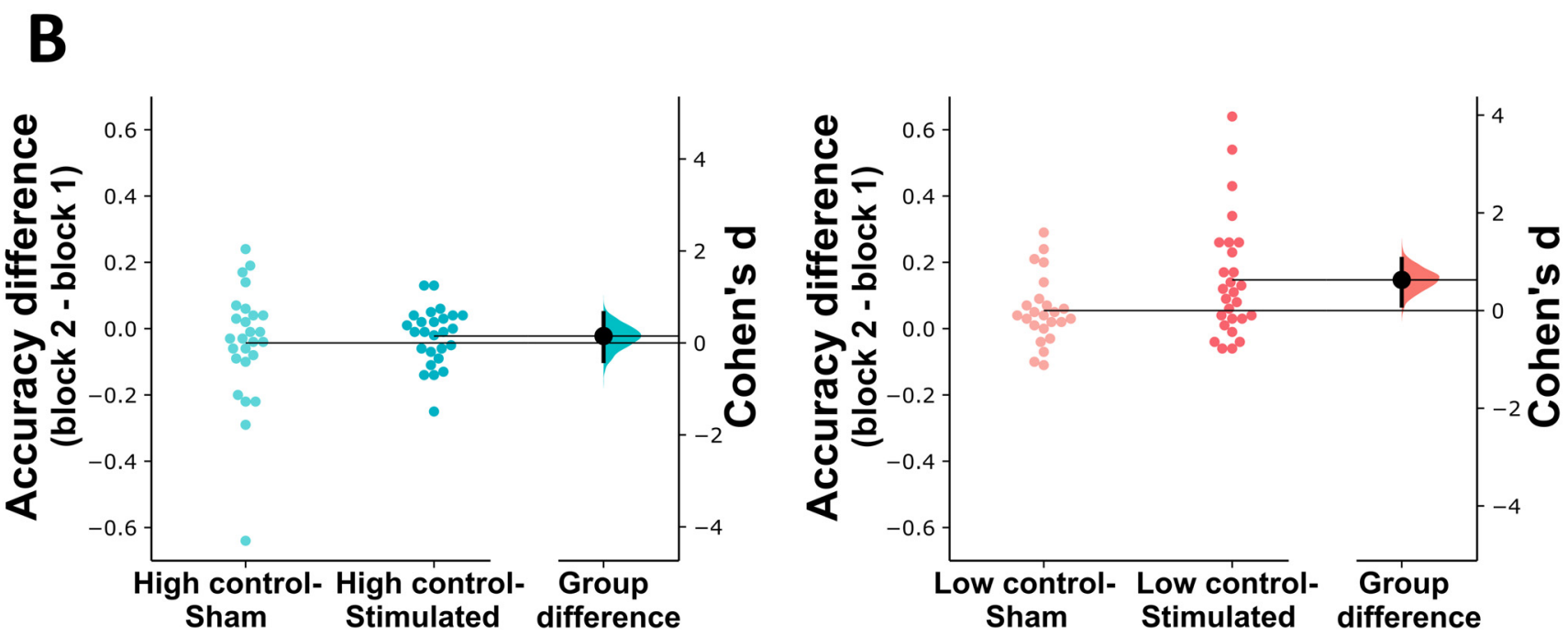

Figure 4. Response accuracy (means and SEs) in each block and experimental group (A). Data corresponding to accuracy for each card type, block and group are presented in Extended Data Figure 4-1. Cumming estimation plots representing effect size estimates (Cohen's $d$ ) for the pairwise comparison of block-effects between groups receiving real versus sham HD-tDCS, calculated separately for HighControl and LowControl groups are shown in $\boldsymbol{B}$. Mean differences are presented as black dots, along with the corresponding bootstrap sampling distributions (5000 samples) and the bias-corrected and accelerated $95 \%$ Cls (black bars). Raw data and Cumming estimation plots related to changes from block 1 to block 2 for each group are presented in Extended Data Figure 4-2. Changes in response accuracy (means and SEs) corresponding to the four groups and Pavlovianconflict versus Pavlovian-congruent cards are plotted separately for Avoid and Win cards (C). Extended Data Figure 4-3 shows the same interaction between card valence, Pavlovian congruency and group, plotted separately for the two blocks. Extended Data Figure 4-4 shows the percentage of Go responses (PercGo) separately for each card, group and experimental block.

Finally, the significant congruency $\times$ valence $\times$ block interaction for response accuracy $\left(F_{(1,99)}=8.89, p=0.004\right.$, $\eta_{\mathrm{p}}{ }^{2}=0.082$ ) was because of improved performance from block 1 to block 2 for NoGo cards only (NoGo-to-Avoid: $p_{\text {Bonferroni }}<0.001$, NoGo-to-Win: $p_{\text {Bonferroni }}=0.036$, Goto-Avoid: $p_{\text {Bonf }}=0.074$, Go-to-Win: $p_{\text {Bonf }}=0.055$ ), albeit this effect was independent of group (four-way interaction: $F_{(3,99)}=0.29, p=0.833, \eta_{p}{ }^{2}=0.009$ ).

\section{Computational modeling}

Modeling in this study was not preregistered, and thus, it should be regarded as exploratory. We extracted four 
A Pavlovian bias $(\pi)$

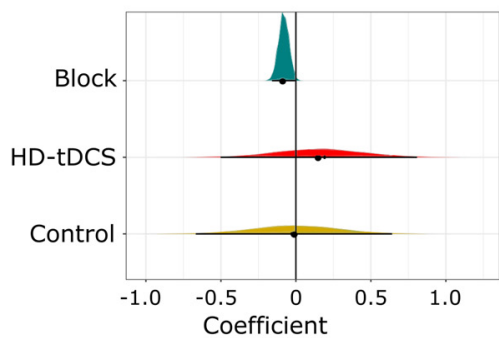

B Learning rate $(\alpha)$

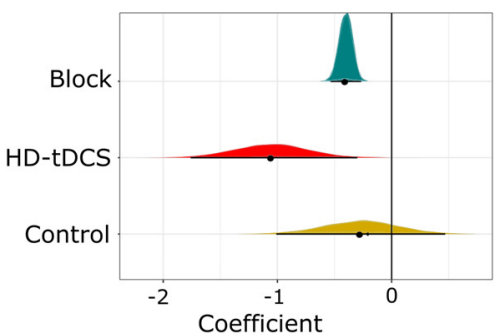

C Temperature(6)

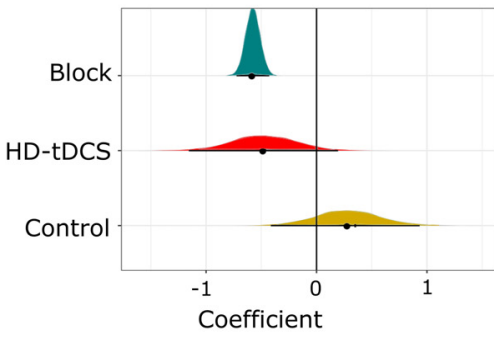

D Go-bias $\left(b_{g o}\right)$

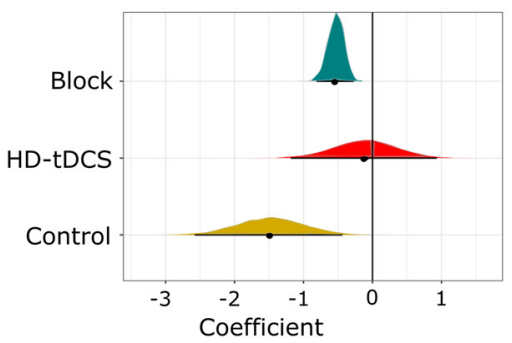

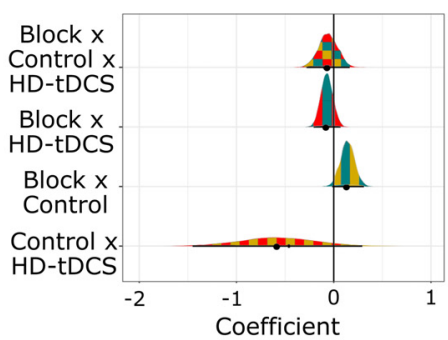
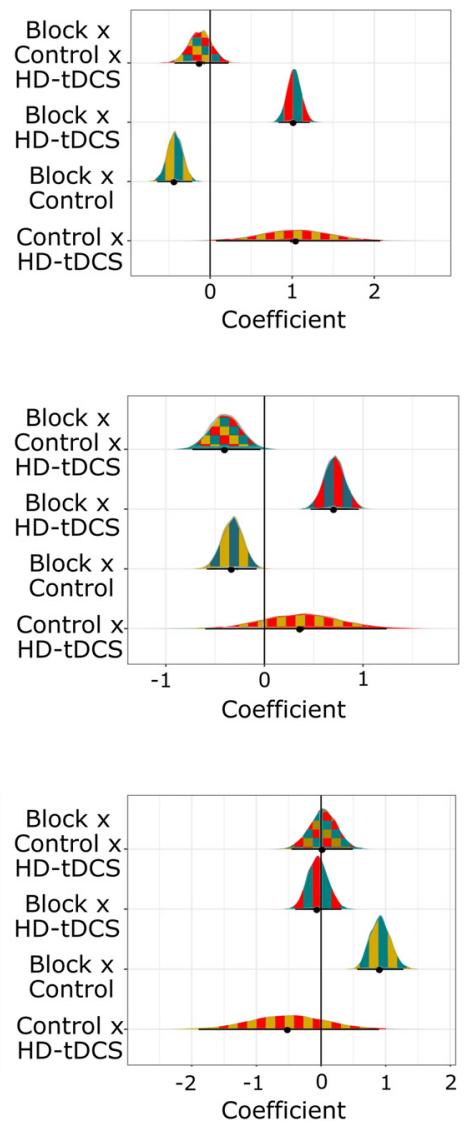

Figure 5. Results from computational modeling. Posterior densities for estimates for the regression coefficients for PB (parameter $\pi ; \boldsymbol{A})$, learning rate (parameter $\alpha ; \boldsymbol{B})$, temperature/randomness of choice (parameter $\beta ; \boldsymbol{C}$ ), and Go-bias (parameter $\left.b_{g o} ; \boldsymbol{D}\right)$.

latent parameters of learning and decision-making: $\mathrm{PB}$ $(\pi)$, learning rate $(\alpha)$, temperature $(\beta)$ and Go-bias $\left(b_{g o}\right)$.

Posterior distributions for the group-level distribution of the Pavlovian parameter were in the positive range $(\pi=$ $0.59,[0.20,0.99])$, confirming that learned stimulus valence biased decisions in the expected manner (action/inaction for positive/negative values, respectively). The estimated $95 \% \mathrm{HDI}$ for the Block coefficient on the PB was negative $(b=-0.08[-0.15,-0.01], P(b<0)=0.993$, $E R_{-}=135.3$ ), in line with results from rmANOVA that PB was partially learnt away by block 2 . Moreover, predominantly positive values for the block $\times$ control interaction $\left(0.14\right.$ [-0.01, 0.29], $P(b>0)=0.974, \mathrm{ER}_{+}=33.3$; Fig. $\left.5 A\right)$ provided evidence for our hypothesis that controllability interfered with reductions in PB throughout the task (i.e., changes in PB from block 1 to block 2 were weaker in LowControl groups). Lastly, we found some support for the interaction between controllability and HD-tDCS (i.e., weaker PB in the LowControl-Stimulated group; $b=$ $\left.-0.58[-1.37,0.17], P(b<0)=0.927, E_{-}=12.7\right)$. Although the result indicated that posterior estimates for the control $\times$ HD-tDCS interaction were 12.7-times more likely to be in the negative range than being either positive or zero (in line with Fig. 3B,C), the effect was not compelling since the $95 \% \mathrm{HDI}$ included zero.

Next, we looked at the learning rate parameter $\alpha$ resembling the degree to which participants updated their stimulus-action values on a trial-by-trial basis. Group-level coefficients were in a similar range as observed in previous reports $(\alpha=0.20,[0.09,0.34])$. Again, we found 
negative values for the Block coefficient, indicating reduced learning rates by block $2(b=-0.40[-0.52$, -0.28], $P(b<0)>0.999$, ER $=\infty$; Fig. 5B). This effect was modulated by controllability and HD-tDCS in the opposite manner, with stronger reduction in LowControl participants (block $\times$ control: $b=-0.43[-0.62,-0.24], P$ $\left.(b<0)>0.999, E_{-}=\infty\right)$, but weaker change following real stimulation (block $\times$ HD-tDCS: $b=1.03$ [0.86, 1.20], $P$ $\left.(b>0)>0.999, \mathrm{ER}_{+}=\infty\right)$. While the main effect of HDtDCS was negative $(b=-1.05$ [-1.69, -0.43$], P$ $(b<0)=0.999$, ER $=749.0)$, positive values for the control $\times$ HD-tDCS interaction coefficient implied that stimulation of mPFC was less effective in reducing learning rates when combined with low controllability $(b=1.05$ $\left.[0.17,1.93], P(b>0)=0.991, \mathrm{ER}_{+}=100\right)$.

Posterior distributions for the temperature parameter $(\beta=2.71[1.75,3.99])$ revealed a negative block-effect $(b$ $=-0.58[-0.71,-0.45], P(b<0)>0.999$, ER $=\infty$; Fig. $5 C)$, which can be interpreted as stronger reliance on learned action weights during response selection in block 2. Similarly to parameter $\alpha$, low controllability intensified (block $\times$ control: $b=-0.32[-0.55,-0.11], \quad P$ $\left.(b<0)=0.998, \mathrm{ER}_{-}=544.4\right)$, while HD-tDCS attenuated this effect (block $\times$ HD-tDCS: $b=0.71$ [0.50, 0.91], $P$ $\left.(b>0)>0.999, \mathrm{ER}_{+}=\infty\right)$. Importantly, participants acted less randomly in block 2 when low controllability was combined with HD-tDCS (block $\times$ control $\times$ HD-tDCS: $\mathrm{b}=-0.39[-0.71,-0.10], P(b<0)=0.994$, ER -170.4$)$. We also found some support for a general reduction in $\beta$ values in both groups with HD-tDCS, although the $95 \%$ HDI did not exclude zero (HD-tDCS: $b=-0.48[-1.07$, 0.09], $P(b<0)=0.948$, ER $=18.2)$.

The fourth parameter, $b_{g o}$, represented Go-bias, the tendency to initiate actions regardless of learned stimulus value. As expected, value estimates were mostly positive $\left(b_{g o}=0.30,[-0.40,0.99]\right)$, although associated with high uncertainty. The coefficient for block was negative $(b=$ $-0.53[-0.78,-0.30], P(b<0)>0.999$, ER $=\infty$; Fig. $5 D)$, indicating improved response accuracy from block 1 to block 2 in NoGo trials (see also Extended Data Fig. 4-1). We found strong evidence for the general reduction in Go-bias in LowControl groups (control: $b=-1.48[-2.35$, -0.46], $P(b<0)=0.999$, ER $=665.7)$, mirroring motor passivity in animal studies of LH (Maier and Seligman, 2016). Importantly, this effect was much stronger in block 1 (block $\times$ control: $b=0.92[0.61,1.23], P(b>0)>0.999$, $\left.\mathrm{ER}_{+}=\infty\right)$, pointing toward weak transfer from controllability manipulation to block 2 , where control over outcomes was regained.

\section{Discussion}

With respect to our first study aim, we expected to observe enhanced PB during and following low outcome controllability under sham stimulation. We postulated that such a result would (1) support earlier findings of similar nature (Dorfman and Gershman, 2019; Csifcsák et al., 2020); and (2) pinpoint our controllability manipulation protocol as a potent experimental intervention for inducing decision-making strategies resembling $\mathrm{LH}$ in healthy adults. However, we could only find partial support for this hypothesis: while preregistered analyses revealed comparable PPI values for the HighControl-Sham and LowControl-Sham groups, the PB parameter from the computational model was modulated in the expected direction (i.e., controllability manipulation attenuated the reduction of PB from block 1 to block 2). The discrepancy between results from the model-free PPI analysis and the model-derived Pavlovian parameter was also reported by another study (Csifcsák et al., 2020), indicating that the two measures differ in their sensitivities to changes in PB under low controllability. While PPI is an aggregated measure that simply reflects the propensity of initiating Go responses exclusively in appetitive trials and withholding actions specifically when facing aversive cues, the Pavlovian parameter takes into account the dynamic learning process, i.e., how card values are learnt from trial-to-trial, and the degree to which this valence-specific knowledge contributes to subsequent choices. Therefore, our study supports the view that the model-derived Pavlovian parameter is a more sensitive measure to manipulations of controllability in this experimental setting.

Low outcome controllability was also associated with stronger NoGo tendencies, indicated by a clearly reduced Go-bias parameter. Therefore, our findings do not resolve the controversy about whether experimental conditions resembling $\mathrm{LH}$-induction influence Pavlovian response tendencies or lead to general behavioral passivity, since both mechanisms were implicated in the current study. Notably, weaker Go-bias during our controllability manipulation could also be because of the presence of the Gocost in our task, since refraining from actions was the optimal strategy for LowControl participants in block 1 to maximize their earnings. Altogether, we cannot state that our protocol for manipulating outcome controllability is potent in inducing decision-making patterns resembling $\mathrm{LH}$. This conclusion is also supported by the absence of a transfer effect regarding response accuracy from block 1 to block 2 (i.e., comparable values for HighControl vs LowControl groups in the final block), and by the similar levels of perceived controllability across groups.

Our controllability manipulation schedule was not optimal for several reasons. First, by keeping the Go-cost, participants could exert some level of control over outcomes, and it also led to imperfect matching in reward/ loss magnitude between HighControl and LowControl groups. Second, reduced control over rewards and losses increased uncertainty around action consequences, and thus, controllability was confounded with predictability (Ligneul, 2021). In this regard, neither our protocol, nor those previously described in the literature (Dorfman and Gershman, 2019; Csifcsák et al., 2020), including the seminal animal studies (Maier and Seligman, 1976), offer a "clean method" of controllability manipulations, with appropriately accounting for changes in outcome predictability. However, a new experimental setting has been introduced recently, that enables manipulations of controllability without confounding it with uncertainty (Ligneul et al., 2020). The authors show that an information-theoretic measure, transfer entropy, can efficiently capture environmental controllability, being dynamically inferred by 
the agent during the task. Future work could test whether other controllability manipulation schedules (similar to the one described by Ligneul et al., 2020) are more effective in inducing LH-like choice behavior in healthy adults.

The second aim of our study was to investigate how HD-tDCS above the mPFC influenced task performance under controllable versus largely uncontrollable response-outcome relationships. Our most striking result is that following low controllability, improvement in response accuracy was stronger in participants receiving HD-tDCS relative to those undergoing sham stimulation. This implies that stimulation above the mPFC/dACC led to more efficient adjustments in decision-making strategies following low controllability, when control over rewards and losses was regained. Although our simulations indicate that HD-tDCS-induced electric fields might have reached the dACC, these modeling results have not been validated by intracranial recordings, and therefore, they should be interpreted with caution. Nevertheless, the mPFC (and dACC in particular) has been associated with conditions of low controllability in the context of LH (Bauer et al., 2003; Diener et al., 2010), but also with tracking changes in environmental volatility (Behrens et al., 2007) and the implementation of CC during various cognitive tasks (Shenhav et al., 2013; Cavanagh and Frank, 2014). Interestingly, a recent study found evidence for trial-by-trial correspondence between frontal midline theta power (an electrophysiological correlate of dACC activity, associated with the implementation of $\mathrm{CC}$ ), and subjectively inferred controllability in a very similar Go/ NoGo task (Gershman et al., 2021). Moreover, anodal tDCS above the rostromedial PFC enhanced gathering information about one's sense of being in control in a social context (Ligneul et al., 2016). Based on these findings, a possible mechanism for the observed effect in our study is that HD-tDCS improved the precision of controllability estimations rather than affecting PB per se. Following that interpretation, the LowControl-Stimulated group could more effectively adjust decision-making strategies to environmental constraints in both blocks. This is apparent in their lower number of Go responses for Win cards in the first block, a behavior that has been associated with reduced exploration tendencies (Teodorescu and Erev, 2014). Withholding Go tendencies was adaptive in this context, since active responses reduced the magnitude of outcomes by the Go-cost, without directly influencing reward/loss frequency. Conversely, in block 2, the LowControl-Stimulated group adjusted their choices in Pavlovian-conflict trials only, a response pattern that has been associated with increased cognitive effort (Cavanagh et al., 2013; Swart et al., 2018). Thus, we conclude that HD-tDCS above the mPFC facilitated LowControl participants' task performance in an adaptive way, possibly via improving their assessment of environmental controllability.

The interaction between controllability and HD-tDCS was most striking in Win trials, resulting in better accuracy for conflicting NoGo-to-Win versus congruent Go-to-Win cards in the LowControl-Stimulated group. Moreover, this group was the only one to show improved responding to
Go-to-Win cards from block 1 to block 2. These observations suggest that only LowControl-Stimulated participants could successfully suppress maladaptive PB in conflicting NoGo-to-Win trials by the end of the task, without overcompensating this strategy at the expense of congruent Go-to-Win cards. Such a selective overcompensation of NoGo response tendencies in Win trials (resulting in a reversal of the congruency effect) was also reported in another study, albeit only for participants with higher levels of outcome controllability (Csifcsák et al., 2020). A possible reason for this discrepancy is that LowControl participants in the study by Csifcsák et al. (2020) were initially exposed to a controllable version of the task, whereas in the current task, they immediately started the task with the manipulated block. Therefore, we propose that prior exposure to controllable outcomes plays a key role in how uncontrollable task-contexts shape decision-making. It remains puzzling, however, why we found stronger HD-tDCS effects for reward-predictive trials. While explanation for this phenomenon awaits future investigation, it is noteworthy that a recent neuroimaging study reported valence-action mappings during the processing of preparatory cues in the ACC for win (but not loss) trials, as well as increased ACC activity during target stimulus presentation for both approach versus avoid and win versus loss stimuli (Hoofs et al., 2021). Therefore, we speculate that this region is more sensitive to evolutionary dominant action requirements in the appetitive domain.

Recent studies suggest that PB during reinforcement learning is regulated by top-down CC mechanisms (Cavanagh et al., 2013; Swart et al., 2018; Csifcsák et al., 2020). An alternative explanation for the observed effect concerning the interaction between HD-tDCS and controllability is that, rather than influencing estimations of one's controllability over the environment, mPFC stimulation directly facilitated the implementation of $\mathrm{CC}$ in Pavlovian-conflict trials. Conflict-associated CC has been successfully modulated via transcranial electric stimulation above midline frontal areas in a Stroop task (To et al., 2018), and in a similar orthogonalized Go/NoGo task (Turi et al., 2020). Thus, it is feasible that the combined effect of low controllability and HD-tDCS on response accuracy in our study is related to enhanced CC and the consequential suppression of PB. In line with this argument, we found improved accuracies for both NoGo-to-Win and Goto-Avoid cards in block 2 for LowControl-Stimulated versus LowControl-Sham participants. Moreover, Cumming estimation plots provided some evidence for weaker PB in the LowControl-Stimulated group, predominantly in block 1 . However, it should be noted that results from the preregistered PPI analysis and the computational modeling approach were not compelling, so this conclusion based on exploratory analyses should be treated with caution.

One could argue that instead of reducing PB for Win cards, HD-tDCS might have promoted NoGo tendencies globally in LowControl-Stimulated participants. While the model-derived Go-bias parameter is inconclusive for the control $\times$ HD-tDCS interaction, our results from PPI and accuracy analyses argue against this interpretation. First, 
a possible general facilitation of NoGo responding should have influenced the two PB sub-measures (reward-based invigoration and punishment-based suppression) in the opposite direction, but this effect was not present in our results. More specifically, the predisposition for NoGo responses in the LowControl-Stimulated group was not present in Avoid trials (producing even more Go responses in Go-to-Avoid trials in block 2, compared with LowControl-Sham participants) which points toward the specificity of NoGo-enhancement to Win trials. In line with the PB account, Ly and colleagues have reported reduced "affective biasing" of instrumental responding following cathodal tDCS above the frontopolar cortex (Ly et al., 2016). In that study, approach primed by emotional stimuli (roughly equivalent to our Go trials) was faster for appetitive versus aversive cues during sham stimulation, but this effect was reversed in the cathodal condition. Given the diffuse cortical distribution of tDCS-induced electric fields in that protocol (with the return electrode placed above the occipital area), it is possible that the similar finding of weaker PB following tDCS in the two studies was mediated by excitability changes in overlapping cortical areas.

The fact that HD-tDCS did not influence response accuracy in HighControl participants is a surprising finding, as we expected that the HighControl-Stimulated group would show more efficient CC and improved task performance in Pavlovian-conflict trials. It is possible that our controllability manipulation changed neural excitability in target regions so that they became more susceptible to HD-tDCS-induced electric fields. Indeed, behavioral consequences of tDCS are sensitive to dynamic fluctuations in neural activity during stimulation ("state dependency"; Tremblay et al., 2014; Dubreuil-Vall et al., 2019), and a recent study implementing a similar low controllability protocol reported altered neurophysiological responses arising from the mPFC/dACC in LowControl subjects (Csifcsák et al., 2020). Based on these results, we speculate that HD-tDCS in our study was not potent enough to influence task performance in participants with sufficient levels of outcome controllability, whereas controllability manipulation might have lowered the threshold for HDtDCS effects to develop.

Computational modeling revealed two additional latent processes that were influenced by our interventions. While earlier work reported stronger reliance on immediate feedback (i.e., increased learning rates) under reduced outcome controllability (Csifcsák et al., 2020), we found such an effect only when controllability was combined with HD-tDCS. Similarly, there was no conclusive evidence for increased exploration/randomness during choice selection in the LowControl groups, which is in contrast with previous findings (Csifcsák et al., 2020). However, controllability and HD-tDCS exerted opposite (intensifying and attenuating) effects on the reduction of both parameters from block 1 to block 2 . Although we hesitate to provide an interpretation for these interactions as they stem from an exploratory analysis, the results suggest that evaluation of outcome controllability converges with both the rate of feedback learning and randomness of choice in the mPFC/dACC.
Our key finding is that HD-tDCS facilitated task performance in the LowControl group only, an effect that was stronger in the appetitive domain, and possibly related to more precise estimations of controllability and/or to enhanced CC over Pavlovian response tendencies. Moreover, controllability and HD-tDCS showed interactive effects in the gradual accumulation of stimulus-action values and in the tendency to act randomly rather than to rely on reinforcement history. Overall, these results highlight the potential of our protocol for interfering with choice arbitration under low controllability of environmental events, resulting in more adaptive behavior.

\section{References}

Amat J, Baratta MV, Paul E, Bland ST, Watkins LR, Maier SF (2005) Medial prefrontal cortex determines how stressor controllability affects behavior and dorsal raphe nucleus. Nat Neurosci 8:365-371.

Bauer H, Pripfl J, Lamm C, Prainsack C, Taylor N (2003) Functional neuroanatomy of learned helplessness. Neuroimage 20:927-939.

Beck AT, Weissman A, Lester D, Trexler L (1974) The measurement of pessimism: the hopelessness scale. $\mathrm{J}$ Consult Clin Psychol 42:861-865.

Behrens TE, Woolrich MW, Walton ME, Rushworth MF (2007) Learning the value of information in an uncertain world. Nat Neurosci 10:1214-1221.

Boayue NM, Csifcsák G, Puonti O, Thielscher A, Mittner M (2018) Head models of healthy and depressed adults for simulating the effects of non-invasive brain stimulation [version 2; referees: 2 approved]. F1000Res 7:704.

Carpenter B, Gelman A, Hoffman MD, Lee D, Goodrich B, Betancourt M, Brubaker M, Guo J, Li P, Riddell A (2017) Stan: a probabilistic programming language. J Stat Softw 76:1-32.

Carver CS, White TL (1994) Behavioral inhibition, behavioral activation, and affective responses to impending reward and punishment: the BIS/BAS scales. J Pers Soc Psychol 67:319-333.

Cavanagh JF, Frank MJ (2014) Frontal theta as a mechanism for cognitive control. Trends Cogn Sci 18:414-421.

Cavanagh JF, Eisenberg I, Guitart-Masip M, Huys Q, Frank MJ (2013) Frontal theta overrides pavlovian learning biases. J Neurosci 33:8541-8548

Csifcsák G, Boayue NM, Puonti O, Thielscher A, Mittner M (2018) Effects of transcranial direct current stimulation for treating depression: a modeling study. J Affect Disord 234:164-173.

Csifcsák G, Melsæter E, Mittner M (2020) Intermittent absence of control during reinforcement learning interferes with Pavlovian bias in action selection. J Cogn Neurosci 32:646-663.

Datta A, Bansal V, Diaz J, Patel J, Reato D, Bikson M (2009) Gyri-precise head model of transcranial direct current stimulation: improved spatial focality using a ring electrode versus conventional rectangular pad. Brain Stimul 2:201-207.

Dayan P, Berridge KC (2014) Model-based and model-free Pavlovian reward learning: revaluation, revision, and revelation. Cogn Affect Behav Neurosci 14:473-492.

Dayan P, Niv Y, Seymour B, Daw ND (2006) The misbehavior of value and the discipline of the will. Neural Netw 19:1153-1160.

Diener C, Kuehner C, Flor H (2010) Loss of control during instrumental learning: a source localization study. Neuroimage 50:717-726.

Dorfman HM, Gershman SJ (2019) Controllability governs the balance between Pavlovian and instrumental action selection. Nat Commun 10:5826-5828.

Dubreuil-Vall L, Chau P, Ruffini G, Widge AS, Camprodon JA (2019) tDCS to the left DLPFC modulates cognitive and physiological correlates of executive function in a state-dependent manner. Brain Stimul 12:1456-1463.

Gelman A, Rubin DB (1992) Inference from iterative simulation using multiple sequences. Statist Sci 7:457-472.

Gelman A, Carlin JB, Stern HS, Rubin DB (2003) Bayesian data analysis. London: Chapman and Hall/CRC Texts in Statistical Science. 
Gershman SJ, Guitart-Masip M, Cavanagh JF (2021) Neural signatures of arbitration between Pavlovian and instrumental action selection. PLoS Comput Biol 17:e1008553.

Guitart-Masip M, Huys QJ, Fuentemilla L, Dayan P, Duzel E, Dolan RJ (2012) Go and no-go learning in reward and punishment: interactions between affect and effect. Neuroimage 62:154-166.

Hershberger WA (1986) An approach through the looking-glass. Anim Learn Behav 14:443-451.

Ho J, Tumkaya T, Aryal S, Choi H, Claridge-Chang A (2019) Moving beyond $P$ values: data analysis with estimation graphics. Nat Methods 16:565-566.

Hoffman MD, Gelman A (2014) The No-U-turn sampler: adaptively setting path lengths in Hamiltonian Monte Carlo. J Mach Learn Res 15:1593-1623.

Hoofs V, Park HR, Vermeylen L, Boehler CN, Krebs RM (2021) Neural underpinnings of valence-action interactions triggered by cues and targets in a rewarded approach/avoidance task. Cortex 141:240-261.

Huys QJM, Eshel N, O'Nions E, Sheridan L, Dayan P, Roiser JP (2012) Bonsai trees in your head: how the Pavlovian system sculpts goal-directed choices by pruning decision trees. PLoS Comput Biol 8:e1002410.

Kerr DL, McLaren DG, Mathy RM, Nitschke JB (2012) Controllability modulates the anticipatory response in the human ventromedial prefrontal cortex. Front Psychol 3:557.

LeDoux J, Daw ND (2018) Surviving threats: neural circuit and computational implications of a new taxonomy of defensive behaviour. Nat Rev Neurosci 19:269-282.

Ligneul R (2021) Prediction or causation? Towards a redefinition of task controllability. Trends Cogn Sci 25:431-433.

Ligneul R, Obeso I, Ruff CC, Dreher JC (2016) Dynamical representation of dominance relationships in the human rostromedial prefrontal cortex. Curr Biol 26:3107-3115.

Ligneul R, Mainen Z, Ly V, Cools R (2020) Stress-sensitive inference of task controllability. bioRxiv 2020.11.19.390393.

Ly V, Bergmann TO, Gladwin TE, Volman I, Usberti N, Cools R, Roelofs K (2016) Reduced affective biasing of instrumental action with tDCS over the prefrontal cortex. Brain Stimul 9:380-387.

Ly V, Wang KS, Bhanji J, Delgado MR (2019) A reward-based framework of perceived control. Front Neurosci 13:65.

Maier SF, Seligman ME (1976) Learned helplessness: theory and evidence. J Exp Psychol Gen 105:3-46.

Maier SF, Seligman MEP (2016) Learned helplessness at fifty: insights from neuroscience. Psychol Rev 123:349-367.

Maier SF, Watkins LR (2005) Stressor controllability and learned helplessness: the roles of the dorsal raphe nucleus, serotonin, and corticotropin-releasing factor. Neurosci Biobehav Rev 29:829841.

Peirce JW (2007) PsychoPy-psychophysics software in Python. J Neurosci Methods 162:8-13.

Pryce CR, Azzinnari D, Spinelli S, Seifritz E, Tegethoff M, Meinlschmidt G (2011) Helplessness: a systematic translational review of theory and evidence for its relevance to understanding and treating depression. Pharmacol Ther 132:242-267.

Rahman A, Reato D, Arlotti M, Gasca F, Datta A, Parra LC, Bikson M (2013) Cellular effects of acute direct current stimulation: somatic and synaptic terminal effects. J Physiol 591:2563-2578.

Rangel A, Camerer C, Montague PR (2008) A framework for studying the neurobiology of value-based decision making. Nat Rev Neurosci 9:545-556.

Shenhav A, Botvinick MM, Cohen JD (2013) The expected value of control: an integrative theory of anterior cingulate cortex function. Neuron 79:217-240.

Swart JC, Frank MJ, Määttä JI, Jensen O, Cools R, den Ouden HE (2018) Frontal network dynamics reflect neurocomputational mechanisms for reducing maladaptive biases in motivated action. PLoS Biol 16:e2005979.

Teodorescu K, Erev I (2014) Learned helplessness and learned prevalence: exploring the causal relations among perceived controllability, reward prevalence, and exploration. Psychol Sci 25:18611869.

Thielscher A, Antunes A, Saturnino GB (2015) Field modeling for transcranial magnetic stimulation: a useful tool to understand the physiological effects of TMS? Annu Int Conf IEEE Eng Med Biol Soc 2015:222-225.

To WT, Eroh J, Hart J, Vanneste S (2018) Exploring the effects of anodal and cathodal high definition transcranial direct current stimulation targeting the dorsal anterior cingulate cortex. Sci Rep 8:4454-4416.

Tremblay S, Lepage JF, Latulipe-Loiselle A, Fregni F, Pascual-Leone A, Théoret H (2014) The uncertain outcome of prefrontal tDCS. Brain Stimul 7:773-783.

Turi Z, Mittner M, Lehr A, Bürger H, Antal A, Paulus W (2020) Thetagamma cross-frequency transcranial alternating current stimulation over the trough impairs cognitive control. eNeuro 7: ENEURO.0126-20.2020.

Turner ML, Engle RW (1989) Is working memory capacity task dependent? J Mem Lang 28:127-154.

Watson D, Clark LA, Tellegen A (1988) Development and validation of brief measures of positive and negative affect: the PANAS scales. J Pers Soc Psychol 54:1063-1070. 